\title{
The LHCb Vertex Locator and Displaced Vertex Trigger
}

\author{
Chris Parkes ${ }^{1}$ \\ on behalf of the LHCb Collaboration \\ European Laboratory for Particle Physics (CERN), CH-1211, Geneva 23, Switzerland
}

\begin{abstract}
The vertex locator of the LHCb experiment must provide precise information on the production and decay vertices of b-hadrons both off-line and for the second level trigger. This article reviews the design of the vertex detector and vertex trigger algorithm. Recent test beam results are provided and the future development programme is outlined.
\end{abstract}

\footnotetext{
${ }^{1}$ Email: Chris.Parkes@CERN.CH
} 


\section{Introduction}

The LHCb experiment is a single-arm spectrometer with a polar angle coverage of approximately $10 \mathrm{mrad}$ to $300 \mathrm{mrad}$ : it is described in detail elsewhere in these proceedings (1). The vertex locator is a critical component of this detector. The ability to separate B meson decay vertices from the primary interaction point is exploited in the trigger strategy as well as in off-line reconstruction.

In off-line reconstruction (triggering) the vertex locator provides an accuracy of $40 \mu \mathrm{m}(80 \mu \mathrm{m})$ along the beam axis on the primary vertex. $\mathrm{B}_{\mathrm{s}}$ studies, in particular, demand excellent proper time resolution: a resolution of $43 \pm 2 \mathrm{fs}$ is obtained in the $\mathrm{B}_{\mathrm{s}}^{0} \rightarrow \mathrm{D}_{\mathrm{s}}^{-} \pi^{+}$channel. After one year of running this will allow exclusion at $95 \%$ CL of $\mathrm{x}_{\mathrm{s}}$ up to 91 .

The base-line design presented here is described in further detail in (2). Where physics results are quoted they are are based on a full GEANT3 (3) simulation of the LHCb detector used in conjunction with the PYTHIA (4) event generator.

\section{Geometry and Vacuum Vessel}

The vertex locator and its supporting mechanics are accommodated within a $1.8 \mathrm{~m}$ long section of large diameter $(1.2 \mathrm{~m})$ beam pipe. The sensors are located within a secondary vacuum separated from the primary machine vacuum by a $100 \mu \mathrm{m}$ aluminium foil. An engineering drawing of the vacuum vessel is provided as Figure 1.

The active elements of the detector are a sequence of silicon sensor stations placed transverse to the beam direction, as illustrated in Figure 2. A total of 17 stations are envisaged, displaced along $1 \mathrm{~m}$. The number and distribution of the stations is chosen to ensure that the majority of tracks seen downstream in the spectrometer will transverse at least 3 vertex detector planes, and to account for the $\approx 5 \mathrm{~cm}$ longitudinal spread in the interaction region: only $5 \%$ of tracks in the $\mathrm{B}^{0} \rightarrow \pi^{+} \pi^{-}$channel traverse less than three stations.

After consultation with the LHC machine division an inner radius of the detector structure of $5 \mathrm{~mm}$ from the beam axis has been agreed. This is acceptable during LHC physics collision operation, however the detector material must be retracted by $3 \mathrm{~cm}$ during injection. This is achieved by constructing the detector in two halves, which can be moved apart vertically. The two halves are offset by $2 \mathrm{~cm}$ horizontally; this allows a small overlap of the sensitive areas to be achieved, thus aiding alignment.

The inner component of the detector is a wake-field suppressor. In the baseline solution this consists of a set of aluminium wires running parallel to the beam axis. However, designs and simulations using the vacuum foil for the wake-field suppression also exist. In this scenario the foil would be performing three functions, since it also acts as an RF-shield for the detectors. 


\section{Silicon Sensor Design}

\subsection{Strip Layout}

Each sensor station consist of two detector discs to read pseudo-orthogonal coordinates. One detector has circular strips measuring the $\mathrm{R}$ co-ordinate, while the other reads the $\phi$ co-ordinate. The $\phi$ measuring detector strips are tilted from a pure radial design by $5^{\circ}$, with successive stations being rotated such that $\mathrm{a} \pm 5^{\circ}$ stereo angle is developed. This detector design is illustrated in Figure 3. This strip layout reflects the rotational symmetry of the forward detector geometry and is guided by the development of a fast vertex triggering algorithm (see section 6).

Prototype detectors similar to the original design were tested in a test-beam at CERN in spring '98 and the expected performance obtained: a resolution of six microns was achieved in the inner $40 \mu \mathrm{m}$ pitch region of the detectors.

In the original design each $180^{\circ}$ half station was constructed from three detectors, these three detectors each spanned $61^{\circ}$ : the overlap between these components assisting in the internal alignment of the disc. However, in the new prototypes $180^{\circ}$ half-stations are being fabricated on a single wafer. Furthermore the regions of fixed pitch in the $R$ measuring detector shown in Figure 3 are now replaced by a continuously varying pitch: optimising both the resolution and the occupancy. The inner radius of the active area of the silicon has been reduced from $1 \mathrm{~cm}$ to $8 \mathrm{~mm}$.

\subsection{Technology}

The first prototype detectors are a single sided readout $\mathrm{n}^{+}$on $\mathrm{n}$ silicon strip design employing polysilicon biasing, multi guard ring structures and individual p-stop isolation between the strips. However the collaboration is still investigating $\mathrm{p}$ on $\mathrm{n}$ strip designs as smaller pitches and hence improved resolution can be achieved: however the harsh radiation environment favours the more tolerant $\mathrm{n}^{+}$on $\mathrm{n}$ choice.

The detectors are read out using a double-metal technology, as pioneered by DELPHI (5), in which the signal from a given strip is routed out over the top of the other structures via a $1 \mu \mathrm{m}$ thick aluminium strip isolated from the rest of the detector by a thin $\mathrm{SiO}_{2}$ dielectric layer. Thus the readout electronics can be placed outside the acceptance, thereby minimising the multiple scattering. The layout of the routing lines to the readout electronics is constrained by the need to minimise cross-talk and capacitance while preserving the strip order in the readout chain. Maintaining the geometrical strip order facilitates the correction of local common base-line shifts.

\section{Radiation}

At $1 \mathrm{~cm}$ from the beam axis the expected yearly fluence exceeds $1 \times 10^{14}$ $1 \mathrm{MeV} / \mathrm{c}^{2}$ equivalent neutrons, and is dominated by primary charged particles. 
The particle flux in a given detector varies as a function of the radial distance from the beam pipe $(r)$ with a roughly $1 / r^{2}$ dependence. Hence the region of type inverted silicon will expand with collider operation time.

Since the full depletion voltage is proportional to the square of the detector thickness, and this voltage rises rapidly with radiation dose, we have chosen very thin detectors for our base-line solution. 150 micron thick detectors are the preferred option as these are the thinnest detectors that leading suppliers will currently manufacture. Thin detectors have the additional advantages of low current and lower multiple scattering. However, the backplane capacitance is higher and naturally the lower signal has consequences for the required readout chip performance.

Prototype detectors have been irradiated and are currently being tested in the lab, they will be tested in a test-beam in Autumn '99. It is anticipated that the base-line silicon sensor design will be successfully operated in this harsh radiation environment for three years before replacement.

\section{Read-Out System}

\subsection{Front-End Electronics}

The front-end electronics are mounted at approximately $7 \mathrm{~cm}$ from the beam axis. The radiation tolerance of the chips must exceed several hundred krad for one year. If operation is required for the expected lifetime of the detector a rad-hard technology must be used.

Analogue, rather than binary, readout has been chosen since it allows for a better monitoring and control of the radiation damage in the detector. Furthermore it provides better precision of the reconstructed hits and better discrimination between hits and clusters due to electronic noise.

One candidate for the front-end chip is the SCT128A which possesses a fast bipolar analogue front-end and CMOS analogue pipeline and is fabricated in a DMILL technology. However this chip does not meet all the requirements of the vertex detector and is currently being modified. The changes include providing four outputs per chip (one per 32 channels) and decreasing the setup time; these are required to achieve compatibility with the LHCb trigger.

The other option is the design of a new chip, based on the experience of our collaborators with the HELIX. This 'BEETLE' chip is being designed in the .25 $\mu \mathrm{m}$ CMOS process.

A four chip hybrid equipped with SCT128A processors was bonded to the prototype detectors. This was successfully operated with an LHC speed (40 $\mathrm{MHz}$ ) clock in a test-beam in Spring '99, see Figure 5. Using a $300 \mu \mathrm{m}$ thick detector a preliminary result of a respectable signal to Noise ratio of $\approx 20: 1$ was achieved. However, for operation with irradiated $150 \mu \mathrm{m}$ thick detectors some improvement in this value is still required. A signal rise time of 25 ns was measured and after a further $25 \mathrm{~ns}$ the output has reduced to one third of its peak value. Simulation studies on benchmark physics modes show that this fall 
time is acceptable for the second level trigger.

\subsection{Off Detector Electronics}

The data is stored in the front end chip buffer until a decision is obtained from the first level trigger. Analogue information from the 200000 channels is transmitted on 7000 twisted-pair cables through the vacuum tank to the read-out electronics at a distance of about $10 \mathrm{~m}$ from the detector. Following digitisation the data is split into two paths:

- In the first path the data are pre-processed and sent to the second level trigger system (see Section 6). The pre-processing performs pedestal subtraction, common baseline shift correction and the cluster search.

- In the second path the data are stored in a buffer, pending a decision from the second level trigger.

On receiving an accept signal from the trigger the data are transmitted over $60 \mathrm{~m}$, by optical data link, to processing electronics.

\section{Secondary Vertex Trigger}

\subsection{Trigger Overview}

LHCb has a robust four-level trigger strategy, and the vertex detector is involved in all stages. The first level of triggering is based upon the selection of leptons, hadrons and photons with higher than average momentum (several $\mathrm{GeV}$ ) in the plane transverse to the beam direction. It reduces the LHC interaction rate of $40 \mathrm{MHz}$ to an output rate of $1 \mathrm{MHz}$ and has a $4 \mu$ s latency. Two dedicated vertex detector planes are also used at this stage to reject events containing multiple interactions. The second level displaced vertex trigger uses the vertex detector alone and is described below. The third and fourth levels are software triggers. In the third level the displaced vertex trigger is refined using information from the other tracking detectors. Finally it is necessary to reconstruct specific b-hadron decay modes using all the detector components including the RICH.

\subsection{Second Level Trigger}

The second level vertex trigger aims to separate B events from minimum bias events by using the signature of displaced secondary vertices. The input event rate is $1 \mathrm{MHz}$, this corresponds to a data collection rate of $2 \mathrm{~GB} / \mathrm{s}$. The average execution time of the algorithm is not yet fixed but will be in the range $500 \mu \mathrm{s}$ to $1 \mathrm{~ms}$.

A major advantage of the $R-\phi$ geometry of the vertex detector is the assistance it provides in performing a fast second level trigger. Using only the $R$ measuring detectors tracks are constructed in the $R-Z$ projection and can 
be combined to produce two track vertices. The position of the primary vertex may then be determined. $\phi$ detector information is then invoked for all tracks that have a significant impact parameter with respect to the primary vertex. Finally secondary vertices are found that are separated significantly from the primary interaction point.

The performance of this algorithm is shown in Figure 4 for a range of B meson decay channels significant for $\mathrm{CP}$ violation studies. This algorithm has been bench-marked and could be performed on 120 1000 MIP processors.

The second level trigger determines the accuracy with which the detector stations must be positioned and on-line alignment performed. The detectors will be inserted in their mountings with an accuracy of $5 \mu \mathrm{m}$, and the movement of the detectors must be controllable and reproducible to an accuracy of $10 \mu \mathrm{m}$.

The trigger algorithm has been tested on test-beam data. A set of targets was placed in front of a set of 12 prototype detectors. By combining events coming from a particular target an LHCb primary vertex was emulated. Extrapolating to the $\mathrm{LHCb}$ experiment a resolution from the secondary vertex trigger algorithm of $80 \mu \mathrm{m}$ along the beam axis was achieved, in agreement with that expected from the full experiment simulation. By combining the "primary collision event' with an event from the next target a B event was simulated and clear separation between the tracks from the two targets observed.

\section{$7 \quad$ Future Plans}

Test-beam work, further optimisation studies and detailed design work are all currently in progress for the vertex locator. The following major project milestones are foreseen:

- A full half station containing both $R$ and $\phi$ measuring detectors will be bonded to front-end electronics and operated at the LHC beam crossing speed of $40 \mathrm{MHz}$. This will be tested during the year 2000 .

- In early 2001 the vertex locator design will be finalised, and documented in a Technical Design Report.

- Construction of the vertex locator will have commenced by the start of 2003.

- Comprehensive testing of the full assembly is anticipated in 2004 prior to the detector insertion.

- LHCb aims to be ready for the start of LHC running, which is planned for 2005 .

\section{Acknowledgements}

I am grateful for the assistance of my colleagues in the LHCb vertex detector group in the preparation of this presentation and report. 


\section{References}

[1] Olivier Schneider, The LHCb experiment, these proceedings

[2] LHCb Technical proposal, CERN/LHCC 98-4

[3] CERN Program Library Long Writeup W5013 (1993)

[4] T. Sjöstrand, CERN-TH 7112/93 (1993)

[5] V. Chabaud et al., Nucl. Instrum. Methods A368(314) (1996) 


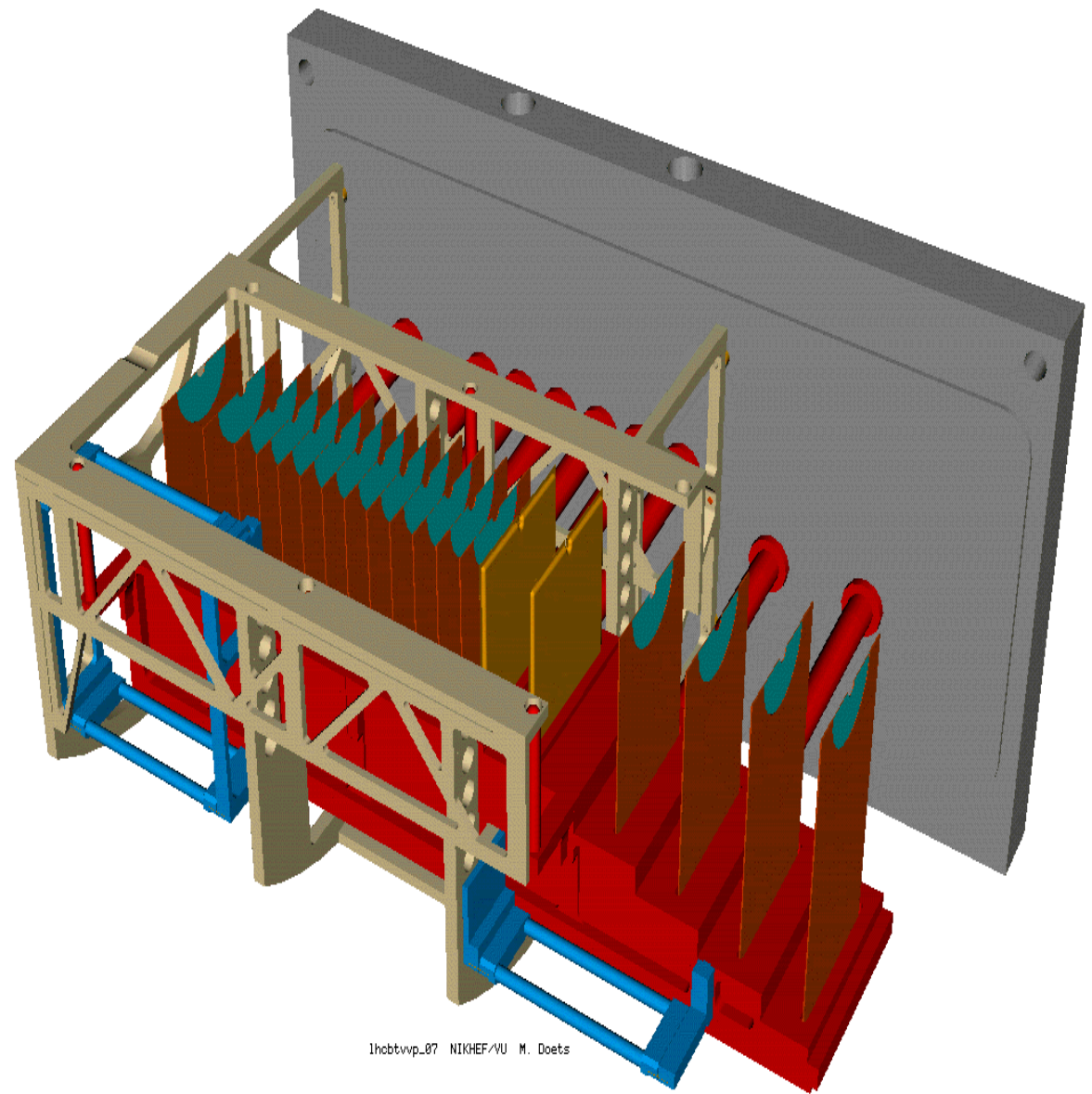

Figure 1: A cut-away diagram of the vacuum vessel. The silicon detectors and mechanics for sensor retraction are visible. 

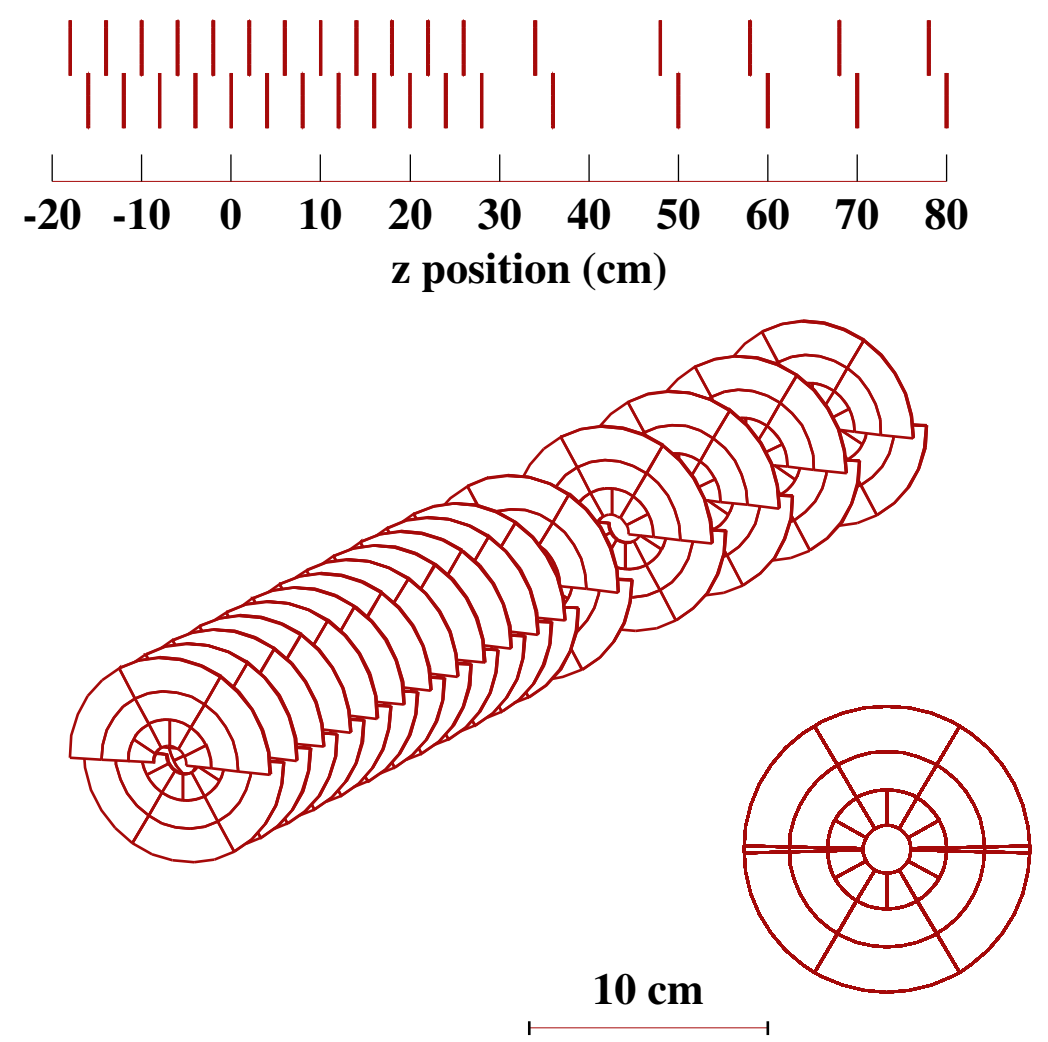

Figure 2: The distribution of the silicon sensor stations along the beam-pipe direction. 


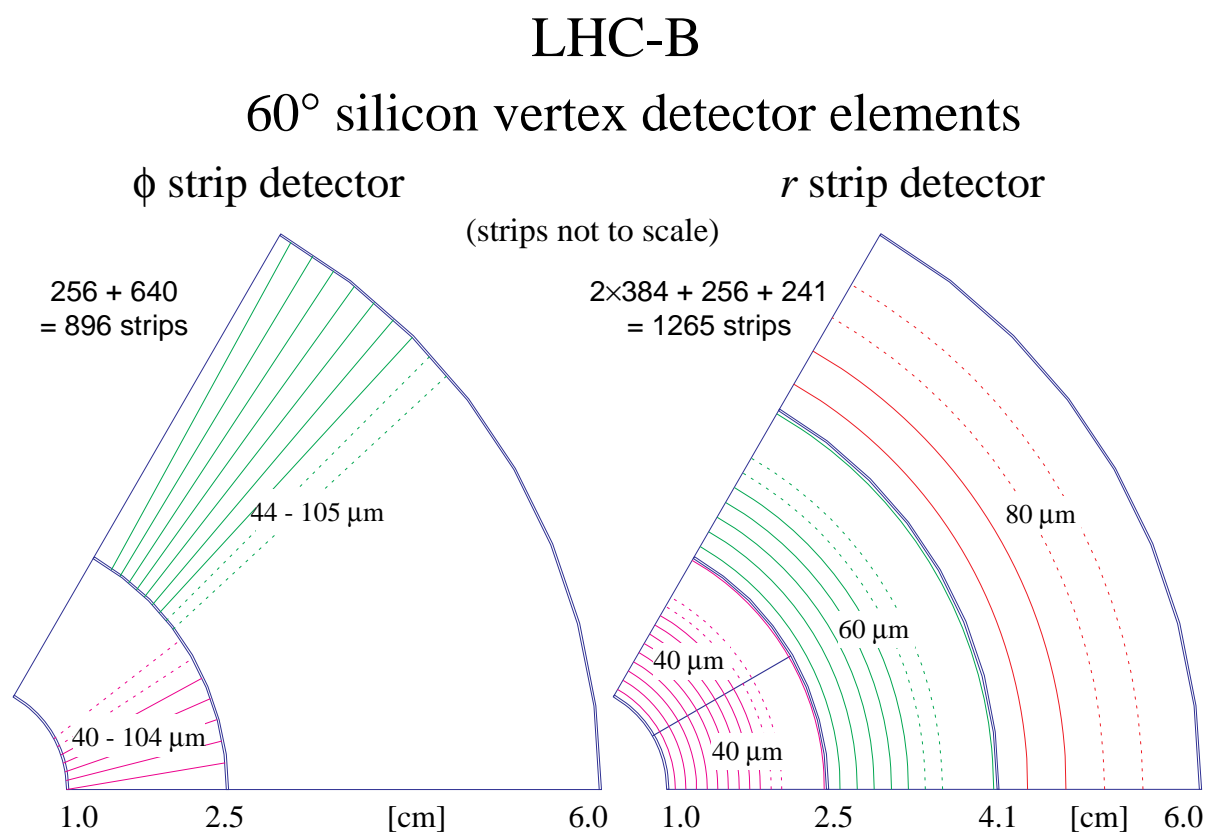

(a)

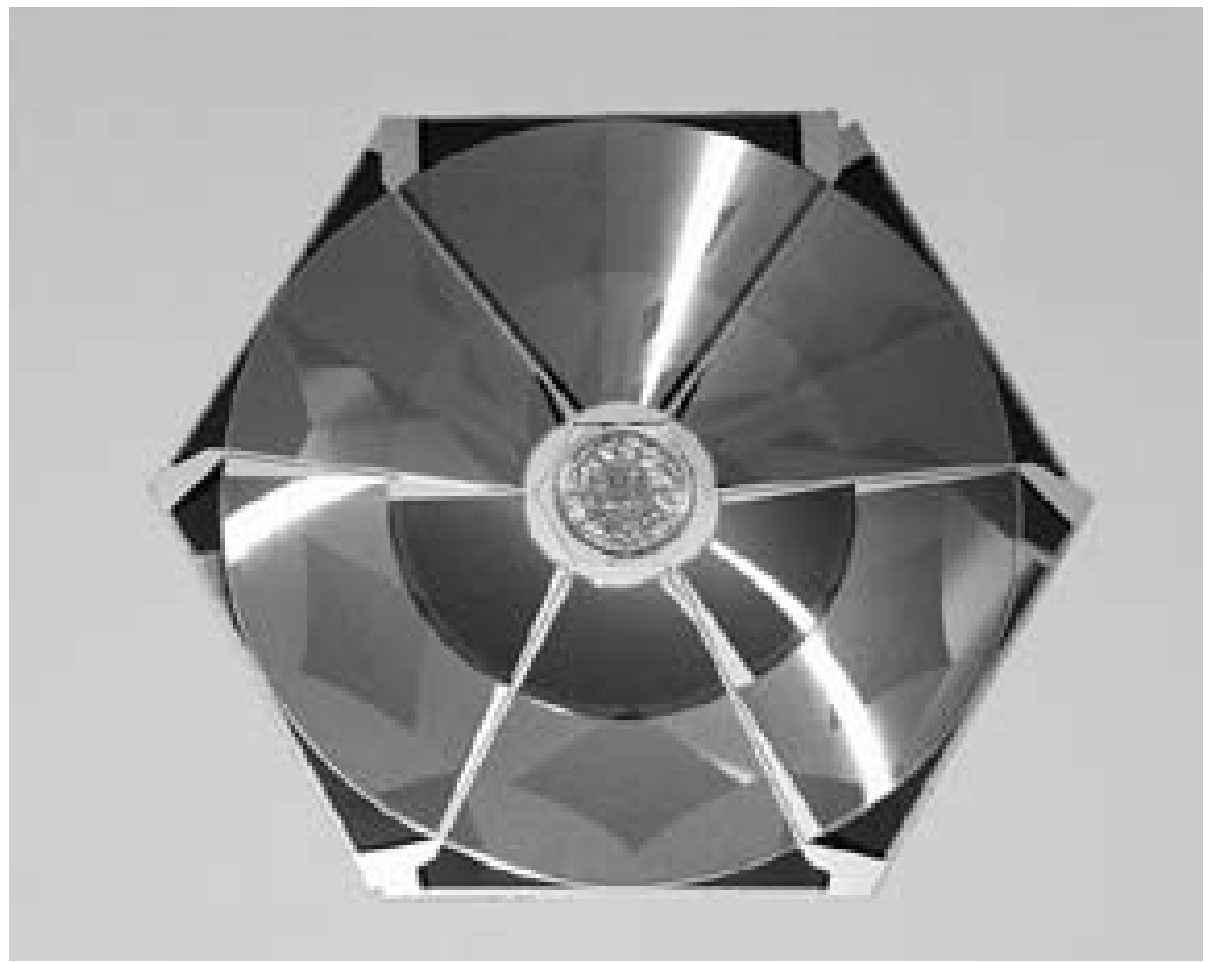

(b)

9

Figure 3: (a) A sketch illustrating the strip layout for the $R$ and $\phi$ measuring detectors. (b) A photograph of the prototype detectors. 


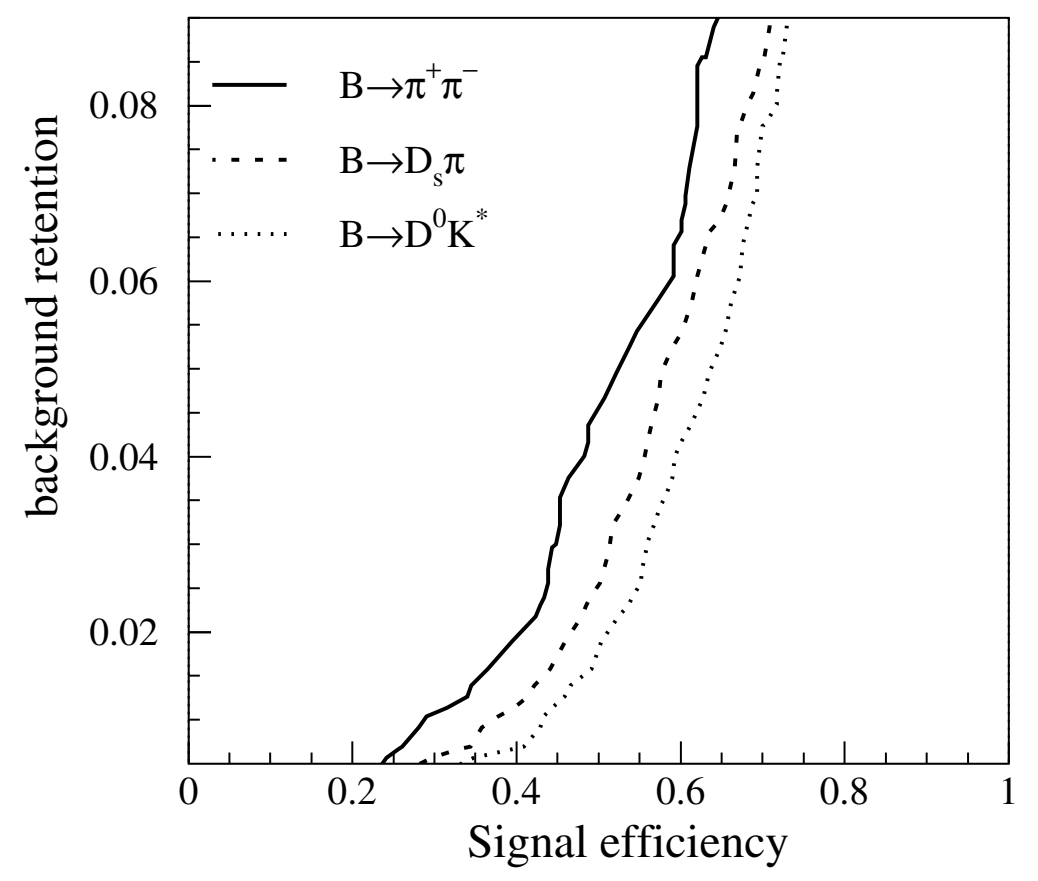

Figure 4: Performance of the displaced vertex trigger algorithm on some standard physics modes. 


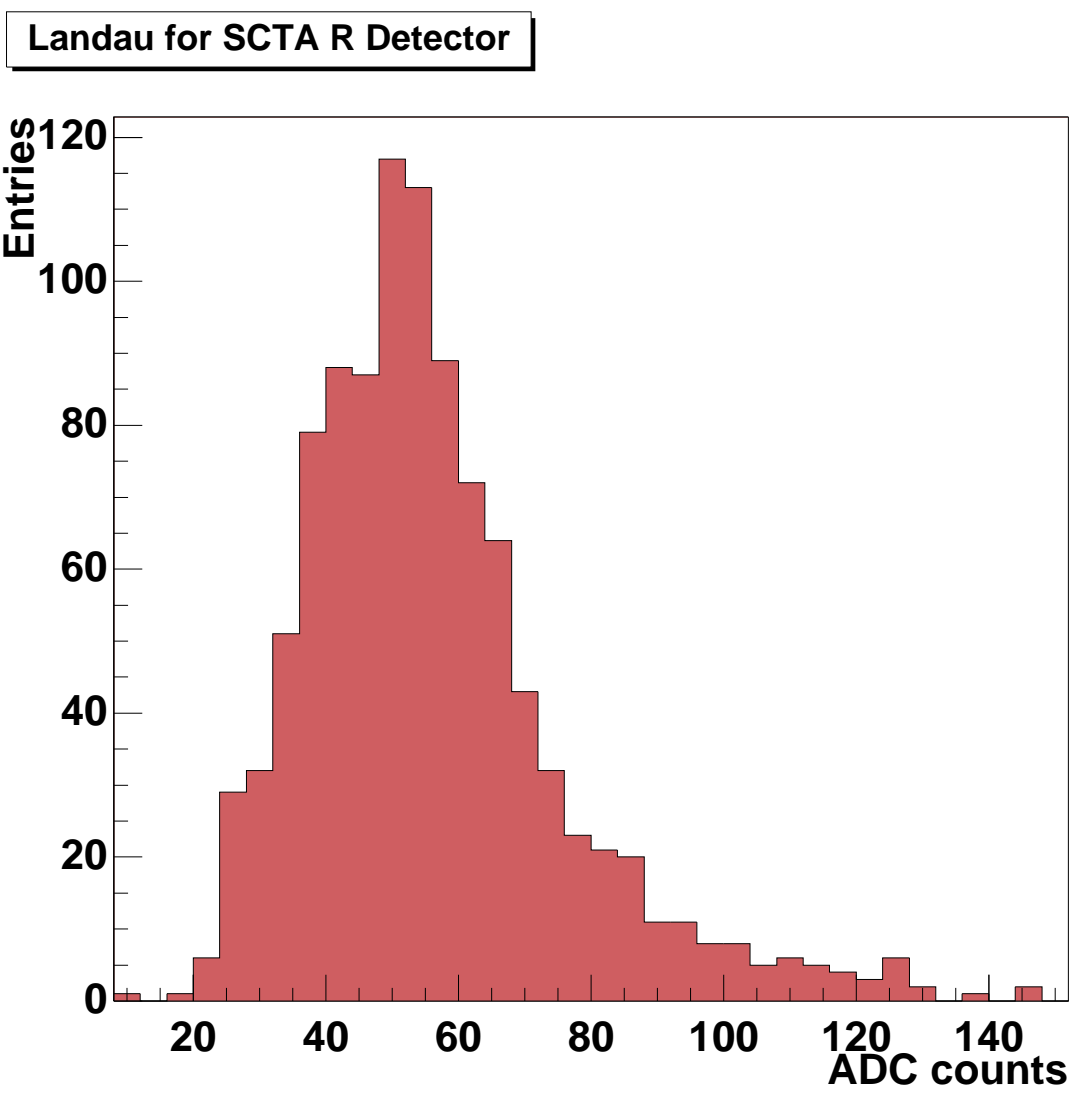

(a)

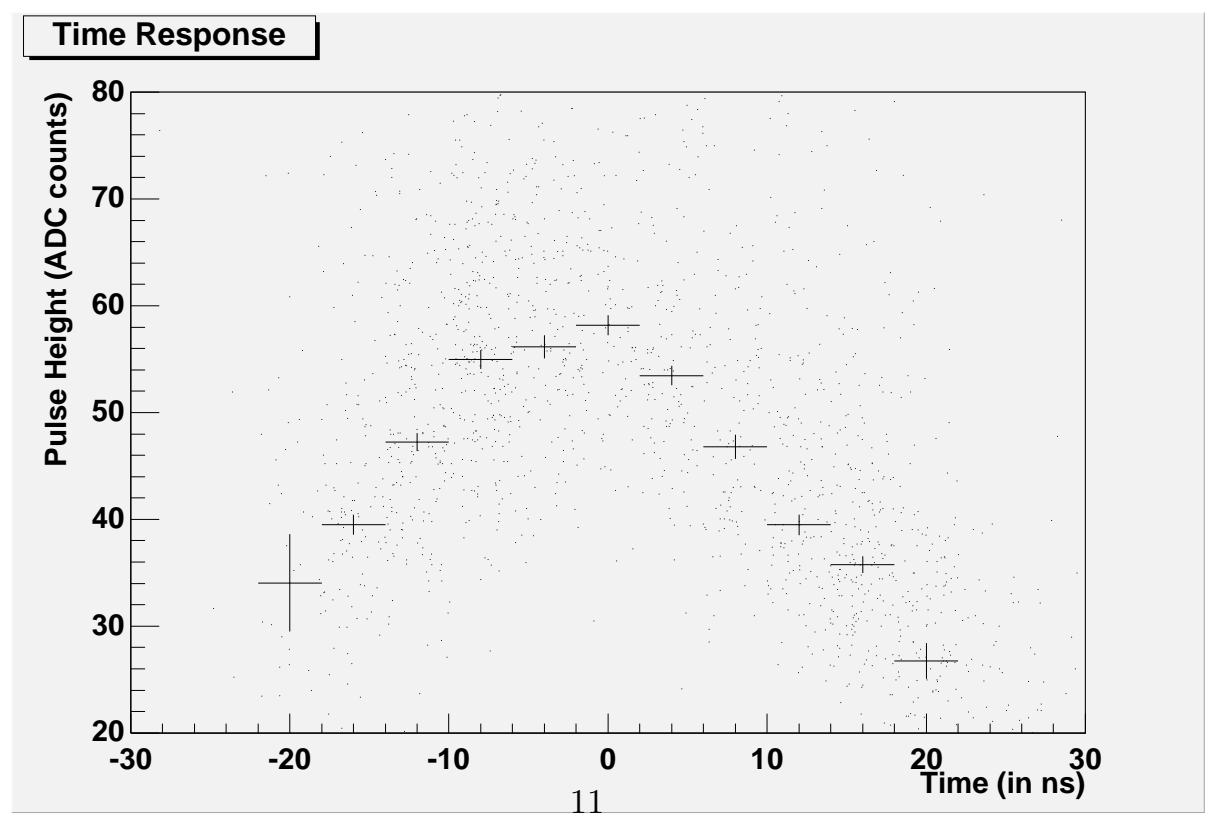

(b)

Figure 5: Preliminary results from the recent test-beam (a) a landau distribution (b) pulse height as a function of time, the crosses correspond to the peak of the landau distribution in each time bin. One ADC count is approximately equivalent to 300 electrons. 